\title{
Voltage/Current-Mode Multifunction Filters Using One Current Feedback Amplifier and Grounded Capacitors
}

\author{
Jiun-Wei Horng, Chun-Li Hou, Wei-Shyang Huang, Dun-Yih Yang \\ Department of Electronic Engineering,Chung Yuan Christian University,Chung-Li, Taiwan, China \\ E-mail: jwhorng@cycu.edu.tw \\ Received November 18, 2010; revised November 22, 2010; accepted November 23, 2010
}

\begin{abstract}
One configuration for realizing voltage-mode multifunction filters and another configuration for realizing current-mode multifunction filters using current feedback amplifiers (CFAs) are presented. The proposed voltage-mode circuit exhibit simultaneously lowpass and bandpass filters. The proposed current-mode circuit exhibit simultaneously lowpass, bandpass and highpass filters. The proposed circuits offer the following features: No requirements for component matching conditions; low active and passive sensitivities; employing only grounded capacitors and the ability to obtain multifunction filters from the same circuit configuration.
\end{abstract}

Keywords: Current Feedback Amplifier, Active Filter, Voltage-Mode, Current-Mode

\section{Introduction}

The current feedback amplifier (CFA) can provide not only constant bandwidth independent of closed-loop gain but also high slew-rate capability. Thus, it is beneficial to use a current feedback amplifier as a basic building block to realize analogue signal processing circuits [1-12].

In 1992 [7], Fabre proposed a voltage-mode bandpass and highpass filters circuit by using two CFAs, one grounded capacitor, one floating capacitor and three resistors. In 1993 [8], Fabre proposed another voltagemode or current-mode biquads. The voltage-mode biquad exhibits simultaneously bandpass and highpass filters by using one CFA, one grounded capacitor, one floating capacitor and two resistors. The current-mode biquad exhibits simultaneously bandpass and highpass filters by using one CFA, two grounded capacitors and two resistors. Several single-CFA voltage-mode biquads were proposed in [9-11]. However, only one filter function (lowpass, bandpass or highpass) can be obtained in each realization, which implies the need to change the circuit topology to obtain other types of filter functions. Moreover, these single-CFA voltage-mode biquads employ floating capacitors. In 1995 [12], Liu proposed four voltage-mode biquads with high input impedance for realization lowpass, bandpass or highpass filters by using two CFAs, two (or three) capacitors and three (or two) resistors. However, only one filter function can be obtained in each realization. More- over, two topologies of Liu's circuits used floating capacitors. In 1996 [10], Soliman proposed many voltage-mode biquadratic filter circuits. The four two-CFA biquads in [10] realize lowpass and bandpass filters simultaneously and using only grounded capacitors.

In this paper, a new configuration is proposed to realize voltage-mode lowpass and bandpass filters simultaneously by using one CFA, two grounded capacitors and three resistors. One more filtering signal can be obtained with respect to the previous single-CFA biquads in [9-11] and two-CFA biquads in [12]. With respect to the voltage-mode biquads in [7-8], the proposed circuit uses only grounded capacitors. The use of grounded capacitors makes the proposed circuit attractive for integrated circuit implementation [13]. With respect to the voltage-mode two-CFA lowpass and bandpass biquads in [10], the proposed circuit uses one less active components.

One new configuration is proposed to realize current-mode lowpass, bandpass and highpass filters simultaneously. One more filtering signal can be obtained with respect to the previous current-mode biquad in [8]. Critical component matching conditions are not required in the design of all proposed circuits.

\section{Voltage-Mode Circuit}

Using standard notation, the port relations of a CFA can 
be characterized by

$v_{x}=v_{y}, v_{o}=v_{z}, i_{z}=i_{x}$ and $i_{y}=0$. The proposed voltage-mode circuit is shown in Figure 1. The output transfer functions of Figure 1 can be expressed as

$$
\begin{gathered}
\frac{V_{l p}}{V_{i n}}=\frac{-G_{1} G_{3}}{s^{2} C_{1} C_{2}+s C_{2}\left(G_{1}+G_{2}+G_{3}\right)+G_{2} G_{3}} \\
\frac{V_{b p}}{V_{\text {in }}}=\frac{s C_{2} G_{1}}{s^{2} C_{1} C_{2}+s C_{2}\left(G_{1}+G_{2}+G_{3}\right)+G_{2} G_{3}}
\end{gathered}
$$

Thus, the circuit realizes an inverting lowpass signal at $V_{l p}$ and a non-inverting bandpass signal at $V_{b p}$, simultaneously. The circuit employs two grounded capacitors, three resistors and only one CFA. Critical component matching conditions are not required. Because the output impedance of the CFA (terminal $v_{o}$ ) is very small, the output terminal of $V_{l p}$ can be directly connected to the next stage. The various parameter values of Figure 1 are given by:

$$
\begin{gathered}
\omega_{o}=\sqrt{\frac{G_{2} G_{3}}{C_{1} C_{2}}}, \frac{\omega_{o}}{Q}=\frac{G_{1}+G_{2}+G_{3}}{C_{1}} \text { and } \\
Q=\frac{1}{G_{1}+G_{2}+G_{3}} \sqrt{\frac{C_{1} G_{2} G_{3}}{C_{2}}}
\end{gathered}
$$

The gain constants are

$$
H_{o(l p)}=-\frac{G_{1}}{G_{2}} \text { and } H_{o(b p)}=\frac{G_{1}}{G_{1}+G_{2}+G_{3}}
$$

One possible design equations for the specified $\omega_{0}$ and $Q$ can be obtained by

$$
\left\{\begin{array}{c}
C_{1}=9 C_{2} Q^{2} \\
G_{1}=G_{2}=G_{3}=3 C_{2} \omega_{o} Q
\end{array}\right.
$$

Under the design Equation (5), the gain constants of Figure 1 become

$$
H_{o(p)}=-1 \text { and } H_{o(b p)}=\frac{1}{3}
$$

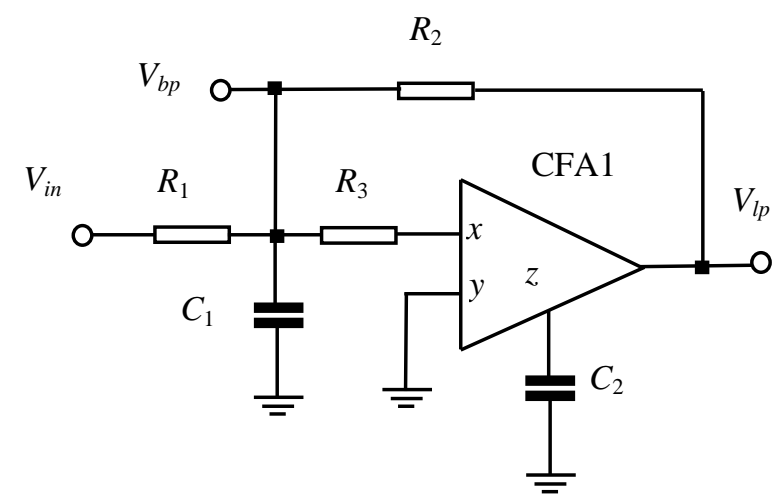

Figure 1. The proposed voltage-mode lowpass and bandpass filter.
All capacitors are grounded in Figure 1. The use of grounded capacitors is particularly attractive for integrated circuit implementation [13]. Moreover, the capacitor $C_{2}$ in Figure 1 is connected to the $\mathrm{z}$ terminals of the CFA, this design offers another feature of a direct incorporation of the parasitic compensation capacitance $\left(C_{p}\right)$ as a part of the main capacitance [14]. Note that, while cascade the bandpass signal of Figure 1, other buffering device is needed because the output impedance of $V_{b p}$ in Figure 1 is not small.

Taking into account the tracking errors of CFA, namely $v_{x}=\beta(s) v_{y}, \quad v_{o}=\gamma(s) v_{z}$ and $i_{z}=\alpha(s) i_{x}$, where $\alpha(s)$ and $\beta(s)$ represent the frequency transfers of the internal current and voltage followers of the CFA, respectively, and $\gamma(s)$ represents the frequency transfer of the output voltage follower of the CFA. They can be approximated by the first order lowpass functions [8]. Assuming the circuits are working at frequencies much less than the corner frequencies of $\alpha(s), \beta(s)$ and $\gamma(s)$, that is, $\beta=1-\varepsilon_{1}$ and $\varepsilon_{1}\left(\left|\varepsilon_{1}\right|<<1\right)$ is the input voltage tracking error, $\gamma=1-\varepsilon_{2}$ and $\varepsilon_{2}\left(\left|\varepsilon_{2}\right|<<1\right)$ is the output voltage tracking error, $\alpha=1-\varepsilon_{3}$ and $\varepsilon_{3}\left(\left|\varepsilon_{3}\right|<<1\right)$ is the current tracking error of a CFA. The resonance angular frequency $\omega_{o}$, bandwidth $\omega_{o} / Q$ and quality factor $Q$ of Figure 1 become

$$
\begin{gathered}
\omega_{o}=\sqrt{\frac{G_{2} G_{3} \alpha_{1} \gamma_{1}}{C_{1} C_{2}}, \frac{\omega_{o}}{Q}=\frac{G_{1}+G_{2}+G_{3}}{C_{1}} \text { and }} \\
Q=\frac{1}{G_{1}+G_{2}+G_{3}} \sqrt{\frac{C_{1} G_{2} G_{3} \alpha_{1} \gamma_{1}}{C_{2}}}
\end{gathered}
$$

The active and passive sensitivities of this filter are

$$
\begin{gathered}
S_{G_{2}, G_{3}, \alpha_{1}, \gamma_{1}}^{\omega_{o}}=-S_{C_{1}, C_{2}}^{\omega_{o}}=\frac{1}{2}, S_{C_{1}, \alpha_{1}, \gamma_{1}}^{Q}=-S_{C_{2}}^{Q}=\frac{1}{2}, \\
S_{G_{1}}^{Q}=-\frac{G_{1}}{G_{1}+G_{2}+G_{3}}, S_{G_{2}}^{Q}=\frac{1}{2}-\frac{G_{2}}{G_{1}+G_{2}+G_{3}}, \\
S_{G_{3}}^{Q}=\frac{1}{2}-\frac{G_{3}}{G_{1}+G_{2}+G_{3}} .
\end{gathered}
$$

All the active and passive sensitivities are no larger than 1.

\section{Current-Mode Circuit}

The proposed current-mode circuit is shown in Figure 2. The output transfer functions of Figure 2 can be expressed as

$$
\begin{aligned}
\frac{I_{l p}}{I_{\text {in }}} & =\frac{-G_{3} G_{4}}{s^{2} C_{1} C_{2}+s C_{2}\left(G_{1}+G_{2}+G_{3}\right)+G_{2} G_{3}} \\
\frac{I_{b p 1}}{I_{\text {in }}} & =\frac{s C_{2} G_{1}}{s^{2} C_{1} C_{2}+s C_{2}\left(G_{1}+G_{2}+G_{3}\right)+G_{2} G_{3}}
\end{aligned}
$$




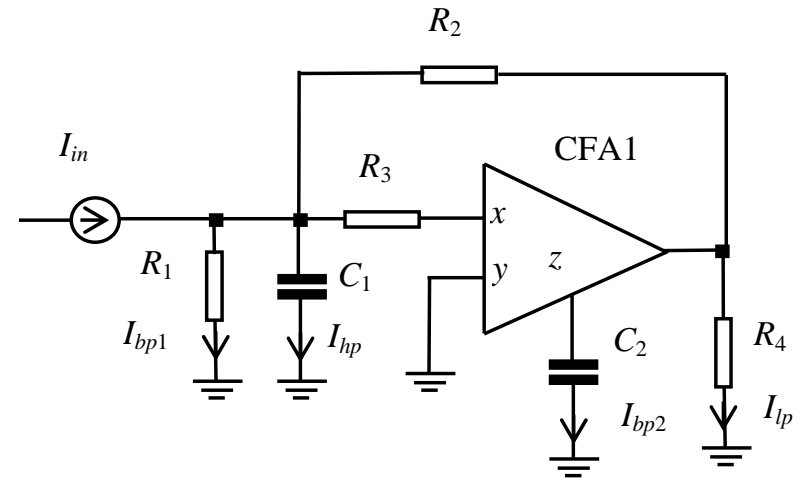

Figure 2. The first proposed current-mode filter.

$$
\begin{aligned}
& \frac{I_{b p 2}}{I_{i n}}=\frac{-s C_{2} G_{3}}{s^{2} C_{1} C_{2}+s C_{2}\left(G_{1}+G_{2}+G_{3}\right)+G_{2} G_{3}} \\
& \frac{I_{h p}}{I_{i n}}=\frac{s^{2} C_{1} C_{2}}{s^{2} C_{1} C_{2}+s C_{2}\left(G_{1}+G_{2}+G_{3}\right)+G_{2} G_{3}}
\end{aligned}
$$

Thus, the circuit realizes an inverting lowpass signal at $I_{l p}$, a non-inverting bandpass signal at $I_{b p 1}$, an inverting bandpass signal at $I_{b p 2}$ and a non-inverting highpass signal at $I_{h p}$, simultaneously. The resonance angular frequency, $\omega_{o}$, bandwidth, $\omega_{o} / Q$, and quality factor, $Q$, have the same values as in Equation (3). The gains of Figure 2 are

$$
\begin{gathered}
H_{o(l p)}=-\frac{G_{4}}{G_{2}}, H_{o(b p 1)}=\frac{G_{1}}{G_{1}+G_{2}+G_{3}}, \\
H_{o(b p 2)}=-\frac{G_{3}}{G_{1}+G_{2}+G_{3}} \text { and } H_{o(h p)}=1
\end{gathered}
$$

\section{Non-ideal Equivalent Circuit of CFA}

The non-ideal equivalent circuit model of the CFA is shown in Figure 3, where $R_{x}$ is the $x$ terminal input resistance, $R_{y} / /\left(1 / s C_{y}\right)$ represents the $y$ terminal parasitic input impedance, $R_{p} / /\left(1 / s C_{p}\right)$ represents the parasitic im- pedance at the compensation terminal $z$ [8]. The typical data sheet values of the various parasitics for the bipolar CFAs (such as AD844) are: $R_{x}=50 \Omega, C_{p}=5.5 \mathrm{pF}$, $R_{p}=3 \mathrm{M} \Omega, \quad R_{y}=2 \mathrm{M} \Omega$ and $C_{y}=2 \mathrm{pF}$. When non-ideal equivalent circuit model of the CFAs are used instead of ideal ones and assuming the circuits are working at frequencies much less than the corner frequencies of $\alpha(s), \beta(s)$ and $\gamma(s)$, namely, $\alpha \cong \beta \cong \gamma \cong 1$, the voltage transfer functions of Figure 1 become

$$
\begin{gathered}
\frac{V_{l p}}{V_{i n}}=\frac{-G_{1} G_{3}{ }^{\prime}}{D(s)} \\
\frac{V_{b p}}{V_{i n}}=\frac{s C_{2}{ }^{\prime} G_{1}+G_{1} G_{p 1}}{D(s)}
\end{gathered}
$$

where

$$
\begin{gathered}
D(s)=s^{2} C_{1} C_{2}{ }^{\prime}+s\left[C_{2}{ }^{\prime}\left(G_{1}+G_{2}+G_{3}{ }^{\prime}\right)+C_{1} G_{p 1}\right]+ \\
G_{2} G_{3}{ }^{\prime}+G_{p 1}\left(G_{1}+G_{2}+G_{3}{ }^{\prime}\right) \\
G_{3}{ }^{\prime}=1 /\left(R_{3}+\mathrm{R}_{\mathrm{X} 1}\right) ; C_{2}{ }^{\prime}=C_{2}+C_{p 1}
\end{gathered}
$$

From Equations (13) to (16), undesirable factors are yielded by the effects of CFA's parasitic impedances. It is found that such factors can be made negligible by operating the filters in high frequencies. But, if the filters are used for lower frequencies, the parasitic impedances could not be negligible. So the characteristics will depart from the theoretical values, especially for the bandpass filter signal in Figure 1. Note that the influence of the parasitic elements on the frequency response of the current-mode filter in Figure 2 can be studies by a similar procedure, as above.

\section{Experimental Results}

Experiments were carried out to demonstrate the feasibility of the proposed circuits. The CFA was imple-
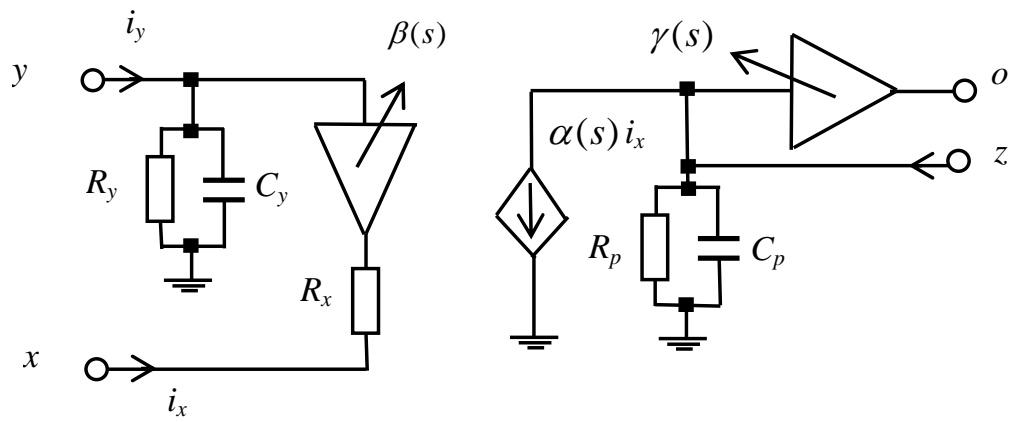

Figure 3. Non-ideal equivalent circuit of the CFA includes the parasitic impedances. 

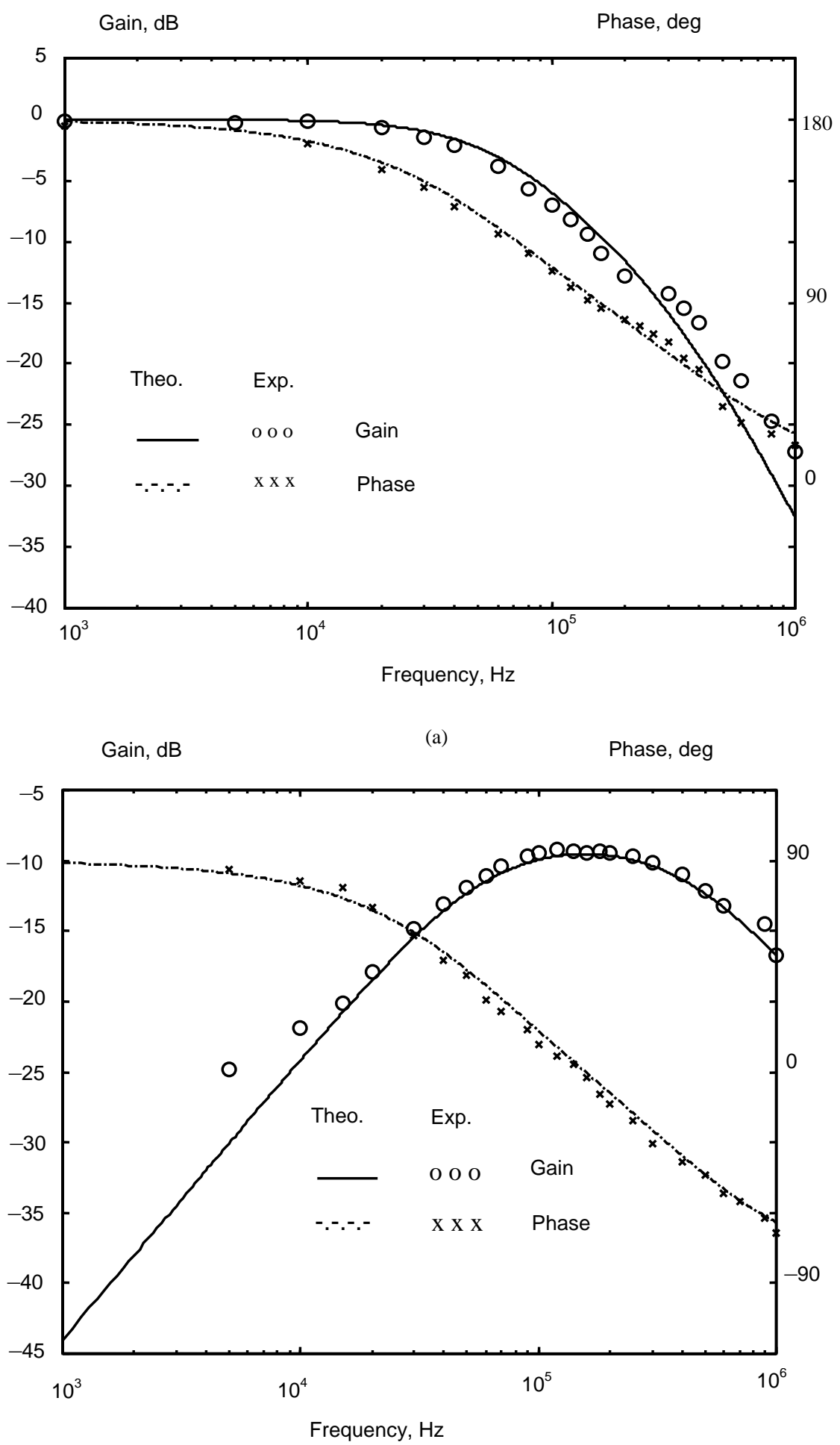

(b)

Figure 4. Experimental frequency responses of Figure 1 design with $C_{1}=C_{2}=100 \mathrm{pF}$ and $R_{1}=R_{2}=R_{3}=10 \mathrm{k} \Omega$. (a) Lowpass filter $\left(V_{l p}\right)$, (b) bandpass filter $\left(V_{b p}\right)$.

mented using one AD844. Figure 4 (a) and (b) represent the frequency responses for the lowpass and bandpass 
filters of Figure 1, respectively, designed with $C_{1}=C_{2}=$ $100 \mathrm{pF}$ and $R_{1}=R_{2}=R_{3}=10 \mathrm{k} \Omega$. Experimental results confirm the theoretical analysis.

\section{Conclusions}

In this paper, a configuration for realizing voltage-mode multifunction filters and a configuration for realizing current-mode multifunction filters using CFAs are presented. The proposed voltage-mode circuit exhibits simultaneously lowpass and bandpass filters by using one CFA, two grounded capacitors and three resistors. The proposed current-mode circuit exhibit simultaneously lowpass, bandpass and highpass filters by using one CFA, two grounded capacitors and four resistors. The proposed circuits have no requirements for component matching conditions. The active and passive sensitivities are low.

\section{References}

[1] S. S. Gupta, D. R. Bhaskar and R. Senani, "New Voltage Controlled Oscillators Using CFOAs," International Journal of Electronics and Communications (AEU), Vol. 63, No. 3, 2009, pp. 209-217. doi:10.1016/j.aeue.2008.01.002

[2] E. Yuce, "Novel Lossless and Lossy Grounded Inductor Simulators Consisting of a Canonical Number of Components," Analog Integrated Circuits and Signal Processing, Vol. 59, No. 1, 2009, pp. 77-82. doi:10.1007/s10470-008-9235-0

[3] R. Nandi, T. K. Bandyopadhyay, S. K. Sanyal and S. Das, "Selective Filters and Sinusoidal Oscillators Using CFA Transimpedance Pole," Circuits, Systems and Signal Processing, Vol. 28, No. 3, 2009, pp. 349-359.

[4] J. W. Horng, P. Y. Chou and J. Y. Wu, "Voltage/Current-Mode Multifunction Filters Using Current-Feedback Amplifiers and Grounded Capacitors," Active and Passive Electronic Components, Vol. 2010, 2010, pp. 1-5. doi:10.1155/2010/785631

[5] K. Kumar and K. Pal, "High Input Impedance Voltage
Mode Universal Biquad Filter,” Indian Journal of Pure and Applied Physics, Vol. 48, No. 4, 2010, pp. 292-296.

[6] N. Nikoloudis and C. Psychalinos, "Multiple Input Single Output Universal Biquad Filter with Current Feedback Operational Amplifiers,” Circuits, Systems and Signal Processing, Vol. 29, No. 6, 2010, pp. 1167-1180. doi:10.1007/s00034-010-9194-4

[7] A. Fabre, "Gyrator Implementation from Commercially Available Transimpedance Operational Amplifiers," Electronics Letters, Vol. 28, No. 3, 1992, pp. 263-264. doi:10.1049/el:19920162

[8] A. Fabre, "Insensitive Voltage-Mode and Current-Mode Filters from Commercially Available Transimpedance Opamps," Proceedings of the Institution of Electrical Engineers, Vol. 140, No. 5, 1993, pp. 319-321.

[9] S. I. Liu and Y. S. Hwang, "Realisation of R-L and C-D Impedances Using a Current-Feedback Amplifier and Its Applications,” Electronics Letters, Vol. 30, No. 5, 1994, pp. 380-381. doi:10.1049/el:19940286

[10] A. M. Soliman, "Applications of the Current Feedback Amplifiers,” Analog Integrated Circuits and Signal Processing, Vol. 11, No. 3, 1996, pp. 265-302. doi:10.1007/BF00240490

[11] A. M. Soliman, "A New Filter Configuration Using Current Feedback Op-Amp,” Microelectronics Journal, Vol. 29, No. 7, 1998, pp. 409-419. doi:10.1016/S0026-2692(97)00025-6

[12] S. I. Liu, "High Input Impedance Filters with Low Component Spread Using Current-Feedback Amplifiers," Electronics Letters, Vol. 31, No. 13, 1995, pp. 1042-1043. doi:10.1049/el:19950725

[13] M. Bhushan and R. W. Newcomb, "Grounding of Capacitors in Integrated Circuits,” Electronic Letters, Vol. 3, No. 4, 1967, pp. 148-149. doi:10.1049/el:19670114

[14] R. Senani, "Realization of a Class of Analog Signal Processing/Signal Generation Circuits: Novel Configurations Using Current Feedback Op-Amps,” Frequenz, Vol. 52, No. 9-10, 1998, pp. 196-206. doi:10.1515/FREQ.1998.52.9-10.196 